\title{
No Treatment for Diabetes
}

National Cancer Institute

\section{Source}

National Cancer Institute. No Treatment for Diabetes. NCI Thesaurus. Code C99994.

No treatment for diabetes. (ACC) 